\title{
Importance of proper diagnosis for management: multifocal choroiditis mimicking ocular histoplasmosis syndrome
}

\author{
Elham Hatef • Peykan Turkcuoglu • Mohamed Ibrahim • Yasir Sepah • \\ Matthew Shulman • Jangwon Heo • Jeong Hee Lee $\cdot$ Roomasa Channa • \\ Afsheen Khwaja • Zubir Rentiya • Syed Mahmood Shah • Diana V. Do • \\ Quan Dong Nguyen
}

Received: 15 September 2010 / Accepted: 9 December 2010 /Published online: 16 March 2011

(C) The Author(s) 2010. This article is published with open access at Springerlink.com

\begin{abstract}
Purpose The study aims to evaluate a series of patients with initial diagnosis of ocular histoplasmosis syndrome (OHS) with progression and response to treatments consistent with multifocal choroiditis (MFC).

Methods Retrospective review of nine patients referred for management of recurrent OHS lesions. Serology panel was conducted to rule out autoimmune and infectious causes. Results Clinical examination revealed multiple small, punched-out peripheral chorioretinal scars, and peripapillary atrophy. Histoplasma antigen/antibody was negative in all patients. Fluorescein angiography and optical coherence tomography confirmed active inflammation in five patients. Immunomodulatory therapy (IMT) was initiated to control active inflammation. While on IMT, visual acuity stabilized or improved in three patients with no recurrence of $\mathrm{CNV}$ or lesion activities over the follow-up period.
\end{abstract}

E. Hatef · P. Turkcuoglu • M. Ibrahim • Y. Sepah • M. Shulman •

J. Heo $\cdot$ J. H. Lee $\cdot$ R. Channa $\cdot$ A. Khwaja $\cdot$ Z. Rentiya $\cdot$

S. M. Shah $\cdot$ D. V. Do $\cdot$ Q. D. Nguyen $(\bowtie)$

Wilmer Eye Institute,

Johns Hopkins University School of Medicine,

600 North Wolfe Street-Maumenee 745,

Baltimore, MD 21287, USA

e-mail: qnguyen4@jhmi.edu

P. Turkcuoglu

Department of Ophthalmology,

Inonu University School of Medicine,

Malatya, Turkey

S. M. Shah

Flaum Eye Institute, University of Rochester School of Medicine,

Rochester,

New York, USA
Conclusions MFC may initially masquerade as OHS. Clinical characteristics of recurrent MFC and absence of histoplasma titer may lead to consideration of IMT and other proper treatments for MFC.

Keywords Multifocal choroiditis · Ocular histoplasmosis syndrome

\section{Introduction}

Differentiating between multifocal choroiditis (MFC) and ocular histoplasmosis syndrome (OHS) may be challenging, especially if based solely on clinical examination. Both may present with chorioretinal (CR) lesions and lack of anterior chamber (AC)/vitreous inflammation [1]. Punchedout chorioretinal scars, peripapillary scarring, and choroidal neovascularization are present in both conditions [2]. However, as the management of OHS differs tremendously from that of MFC, it is important to make proper diagnosis. The index study evaluates a series of patients whose initial diagnosis was OHS. Patients underwent extensive review of systems, uveitis diagnostic survey, a comprehensive examination, and targeted evaluation. The subsequent progression and response to treatments in all patients were consistent with MFC.

\section{Materials and methods}

Nine patients were referred from January 2008 to June 2010 to one of the authors (QDN) for management of OHS. Patients were diagnosed and managed as subjects with OHS 
in their primary institutes; laser photocoagulation/vascular endothelial growth factor (VEGF) antagonists for recurrent choroidal neovascularization (CNV) and episodic prednisone for lesions causing visual disturbances were prescribed. At the Wilmer Eye Institute, serology panel was conducted to rule out autoimmune and infectious causes. Fluorescein angiography (FA) and optical coherence tomography (OCT) were performed at initial and subsequent visits.

The charts of eligible patients were reviewed to include data regarding demographic features of each patient, previous diagnosis of OHS and any concomitant diagnosis, history of any relevant treatment to disease, and the length of follow up. The results of comprehensive ocular examination, serologic workup for all possible inflammatory and infectious conditions, as well as FA and OCT were reviewed. Final diagnosis and treatment applied for each patient was documented as well. Statistical summaries for baseline characteristics were reported. The final diagnosis, treatment regimen, and final outcomes for each patient were presented.

\section{Results}

Eight women and one man with an age range of 2669 years (median: 38 years) were included in the study. Six patients had a previous diagnosis of OHS, two had a likely diagnosis of OHS or punctuate inner choroidopathy (PIC), and one had a questionable diagnosis of uveitis and an inflammatory/infectious process such as sarcoidosis or OHS. Seven of the patients had a history of bevacizumab injection to control CNV, one of whom also received laser photocoagulation and photodynamic therapy; one had photodynamic therapy as well as intraocular injection of triamcinolone acetonide; the other one had photodynamic therapy prior to bevacizumab injection. One patient had a history of only laser photocoagulation to control CNV. The patients were followed up at our institute for a period of 1.18-14.51 months (median; 3.16 months).

We carried out a comprehensive ocular examination as well as serologic workup for all possible inflammatory and infectious conditions based on the clinical findings. Multiple small, punched-out peripheral CR scars and peripapillary atrophy were noted in all patients. No AC/vitreous inflammation was detected. None of them had active CNV at the time of initial visit (Figs. 1 and 2). Histoplasma antigen/antibody for all subjects was performed in the same clinical laboratory through the Johns Hopkins University and Hospitals, and both antigen and antibody were negative in all patients.

Rheumatoid panel and serology for Herpes simplex as well as Herpes zoster were also negative. FA and OCT confirmed active inflammation (choroiditis without CNV) in five patients. Table 1 shows the clinical and imaging findings as well as serologic evaluation for each patient.

Based on the clinical examination and the result of serologic evaluations that were nonrevealing for other causes, a diagnosis of immune-mediated MFC was made in seven patients. Patient 8 had a history of positive PPD testing and ocular finding which might be consistent with tuberculous choroiditis. We recommended the patient to be evaluated for tuberculosis infection as well. For patient 9, based on the clinical findings and negative serology for histoplasmosis, it was most likely that he had CNV, which is idiopathic in nature. There did not seem to be any active $\mathrm{CNV}$ at the time of examination. Therefore, the patient was advised to monitor his vision closely and be re-examined regularly to look for any signs of CNV activity.

Immunomodulatory therapy (IMT) was initiated to control the inflammation in five of the eight patients (patients 1, 2, 5, 6, and 7). They received treatment from 1.84 to 6.22 months. Patient 3 had a complicated medical history of scleroderma, graft-versus-host disease after an allogenic bone marrow transplant for leukemia, history of cytomegalovirus encephalopathy, and shingles. She was on mycophenolate mofetil for scleroderma and graft-versushost disease at the time of initial visit. Considering the underlying hematologic disease, we recommended a consultation with her hematologist/oncologist prior to starting treatment for MFC. For patient 4, based on examination and ancillary testing, it was most likely that she had primary MFC rather than OHS or toxoplasmosis. We decided to monitor the patient and would begin IMT treatment to decrease the risk of recurrent inflammation if active choroiditis or recurrent $\mathrm{CNV}$ reappeared. In addition, VEGF antagonist would be employed as needed to provide rapid control of any recurrent $\mathrm{CNV}$.

While on IMT, visual acuity stabilized or improved in patients 2, 5, and 6 with no recurrence of $\mathrm{CNV}$ or lesion activities over the follow-up period. These three patients were followed up for a period of 3.16, 1.84, and 5.69 months, respectively. Patient 1 had an episode of visual disturbance in the right eye which was accompanied with an enlargement of two previously detected lesions on OCT. The disease activation was not controlled by increase in the dose of IMT; therefore, the patient was enrolled in a clinical trial of local treatment of another IMT. Patient 7 had a worsening of visual acuity in the right eye from 20/ 200 to 20/320 after initiation of IMT (mycophenolate mofetil). Considering the short period of IMT treatment and slow treatment response in the case of IMT, we decided to continue our recommended treatment and follow up the patient every 4 weeks. The visual acuity improved to 20/200, 3 months after initiation of IMT and the dosage was increased to $1.5 \mathrm{~g}$ twice a day at the last visit. Patient 4 and 
Fig. 1 The Optos ${ }^{\mathrm{TM}} \mathrm{P}-200^{\circ}$ digital fundus image of patient 6 illustrates chorioretinal lesions inferiorly in both eyes. Lesions in the left eye are more prominent than those in the right eye. There is a yellowish lesion just inferonasal to foveal center of the left eye

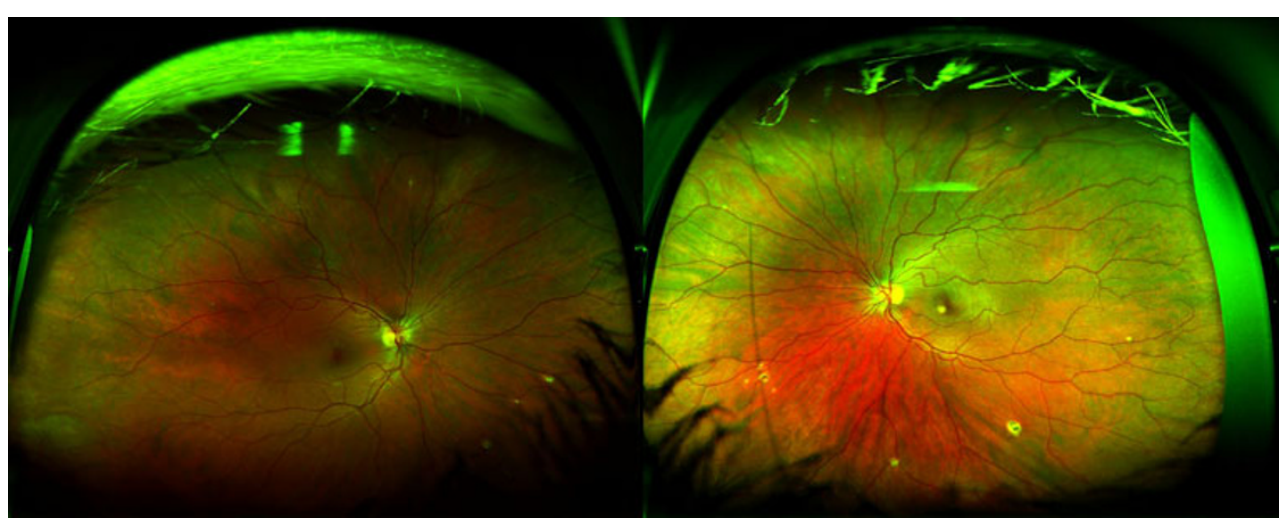

in the amount of fluorescence of the lesions on autofluorescence among those being treated with IMT.

\section{Discussion}

Patients with MFC may masquerade as OHS. The index study suggests that the diagnosis of OHS should not be considered exclusively unless accompanied by positive serology, absence of inflammation, and appropriate clinical course for OHS. Even then, the patients should be followed closely for progression of the lesions as the disease may be of different entities. Our patients had many features that resembled OHS. Peripheral and posterior pole scars like studies of patients with diagnosis of MFC showed decrease

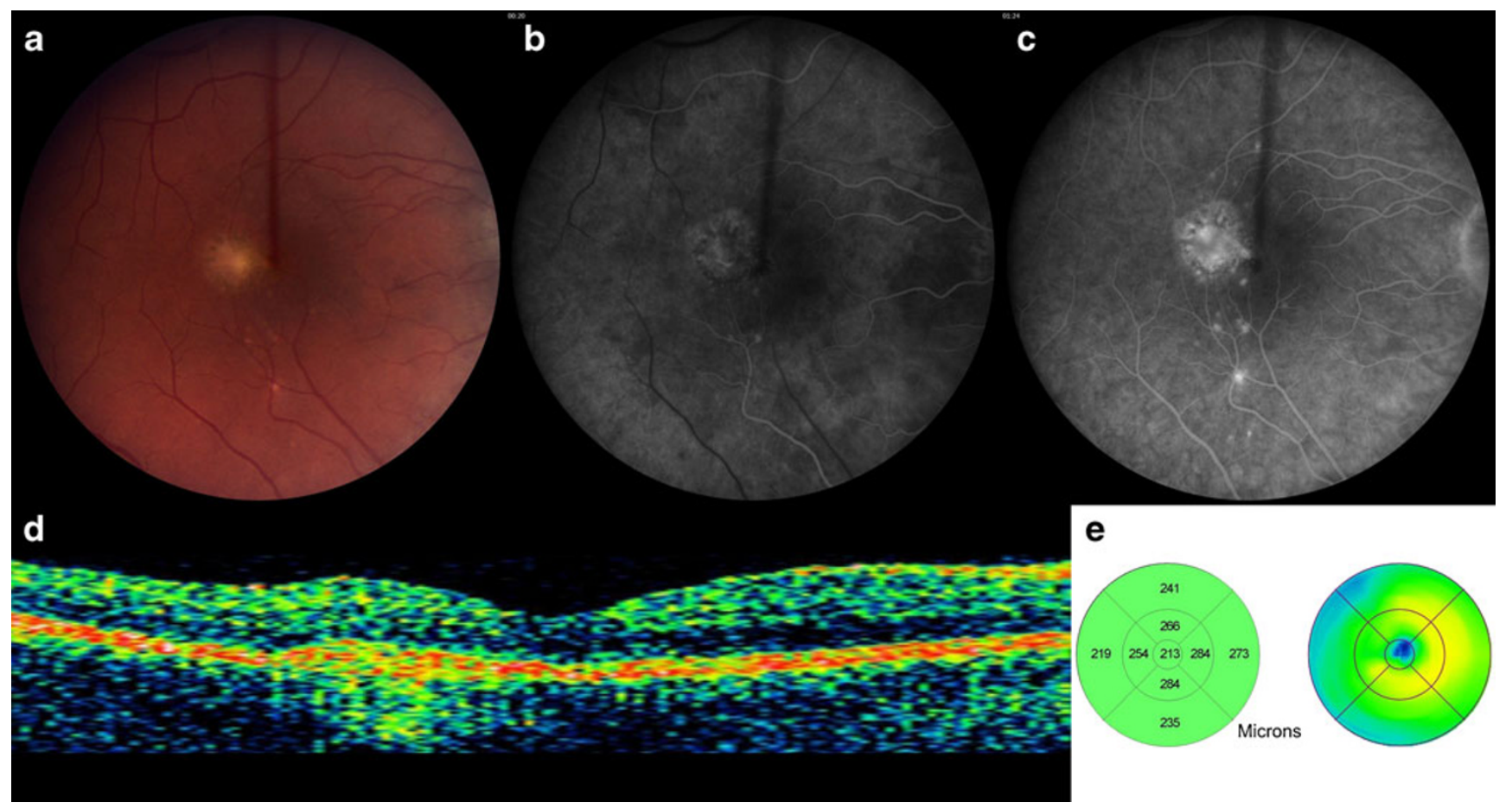

Fig. 2 a Fundus image of the right eye of patient 5 illustrates multiple chorioretinal lesions, which concentrates within the macula. There is a deep chorioretinal scar on the temporal aspect of foveal center with no subretinal fluid. b, c Fluorescein angiography of right eye demonstrates classical choroidal neovascularization (CNV) filling pattern with chorioretinal anastomosis in the center without prominent leakage in early $\mathbf{b}$ and mid $\mathbf{c}$ phases. d, e Optical Coherence Tomography of right eye shows hyperreflective substance within the retinal pigment epithelium (RPE)/choroid complex, with no intraretinal edema 


\begin{tabular}{|c|c|c|}
\hline 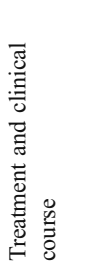 & 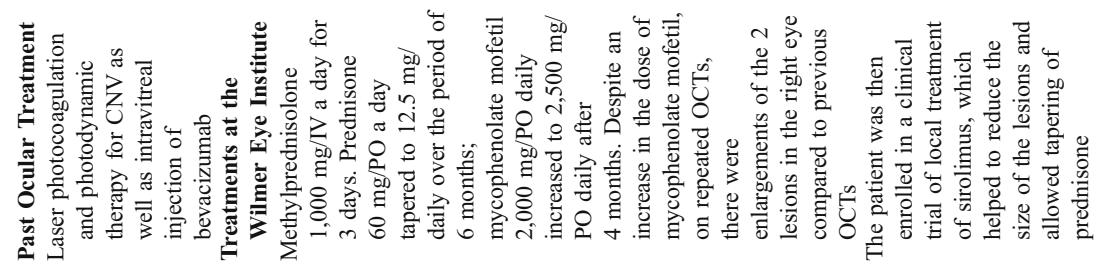 & 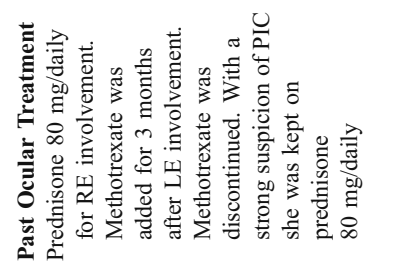 \\
\hline 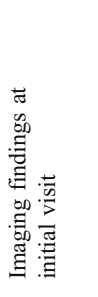 & 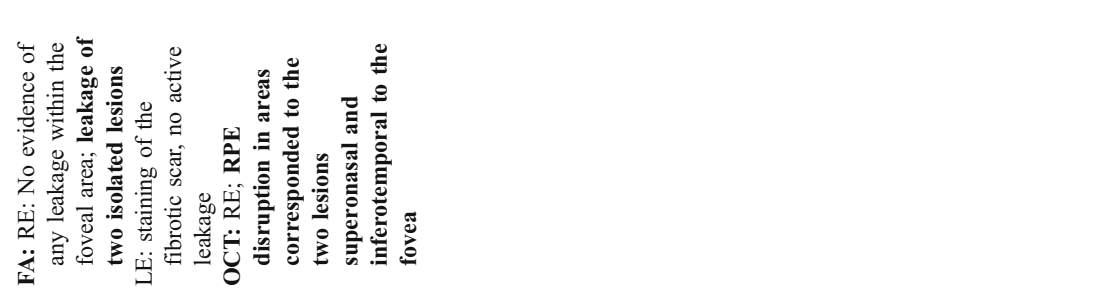 & 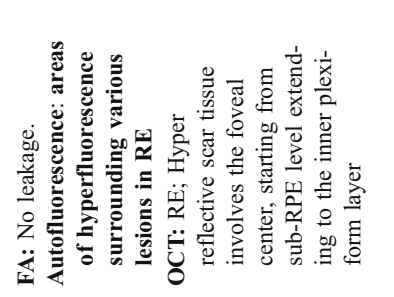 \\
\hline 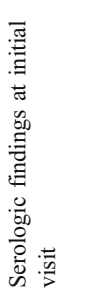 & 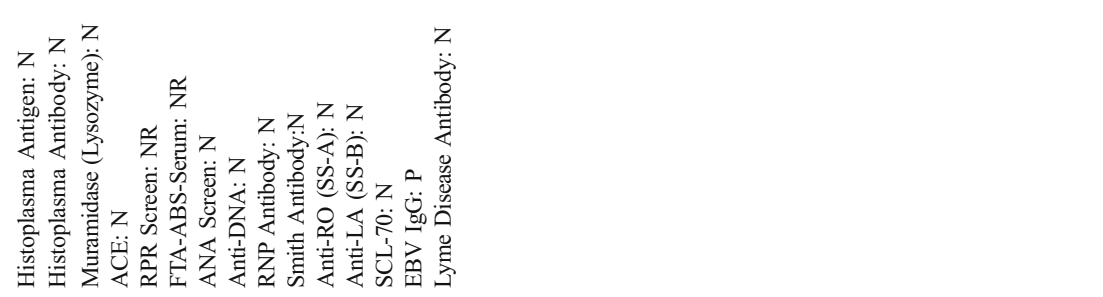 & 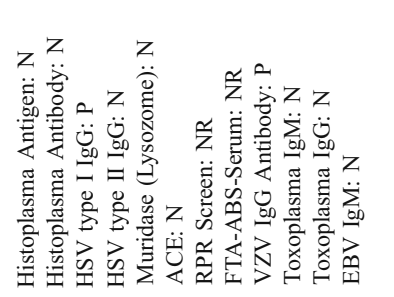 \\
\hline 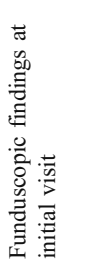 & 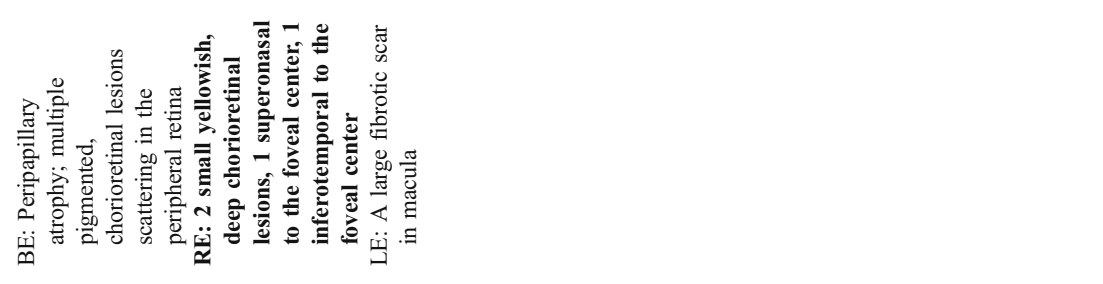 & 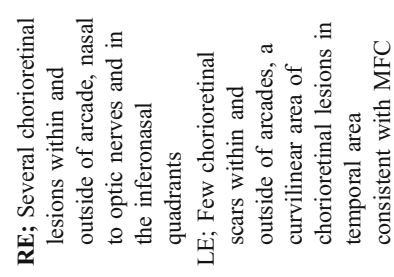 \\
\hline 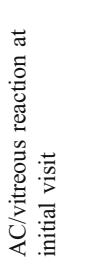 & 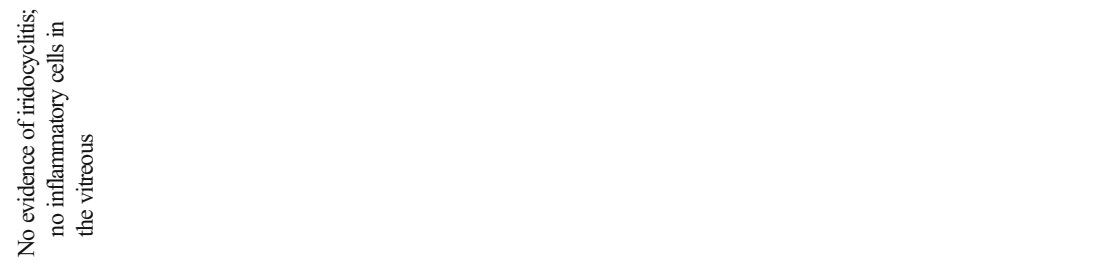 & 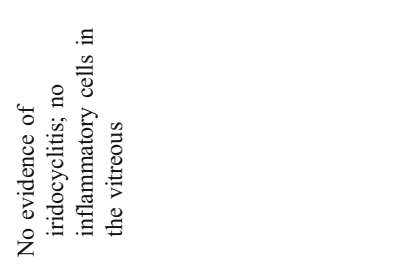 \\
\hline 고 & $\begin{array}{l}\overline{5} \\
\dot{I}\end{array}$ & $\stackrel{\bullet}{m}$ \\
\hline 可 & 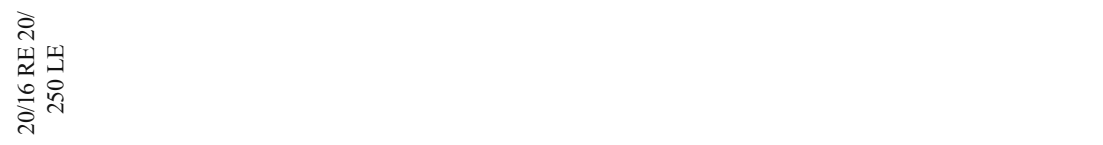 & 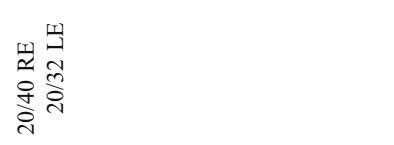 \\
\hline 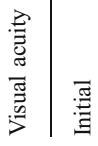 & 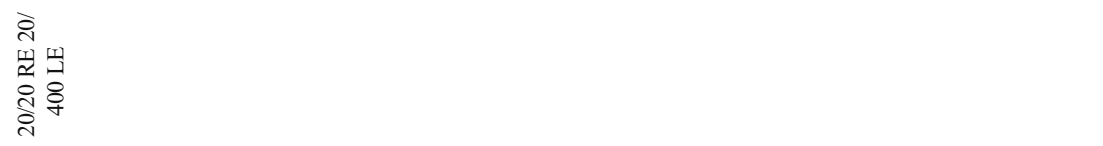 & 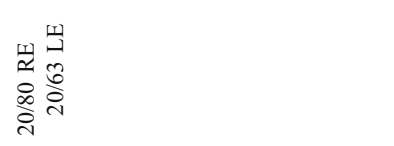 \\
\hline 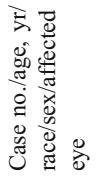 & 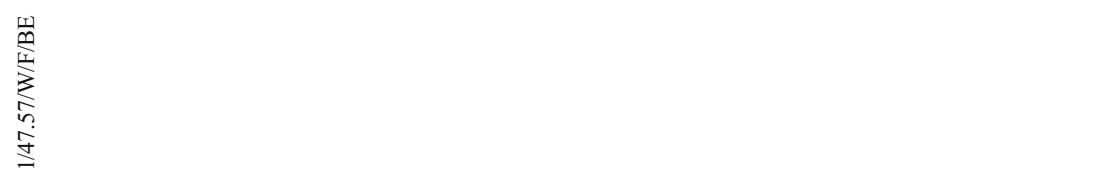 & 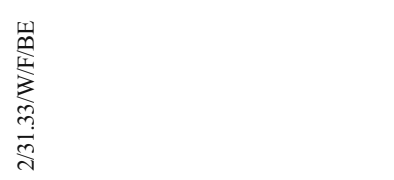 \\
\hline
\end{tabular}



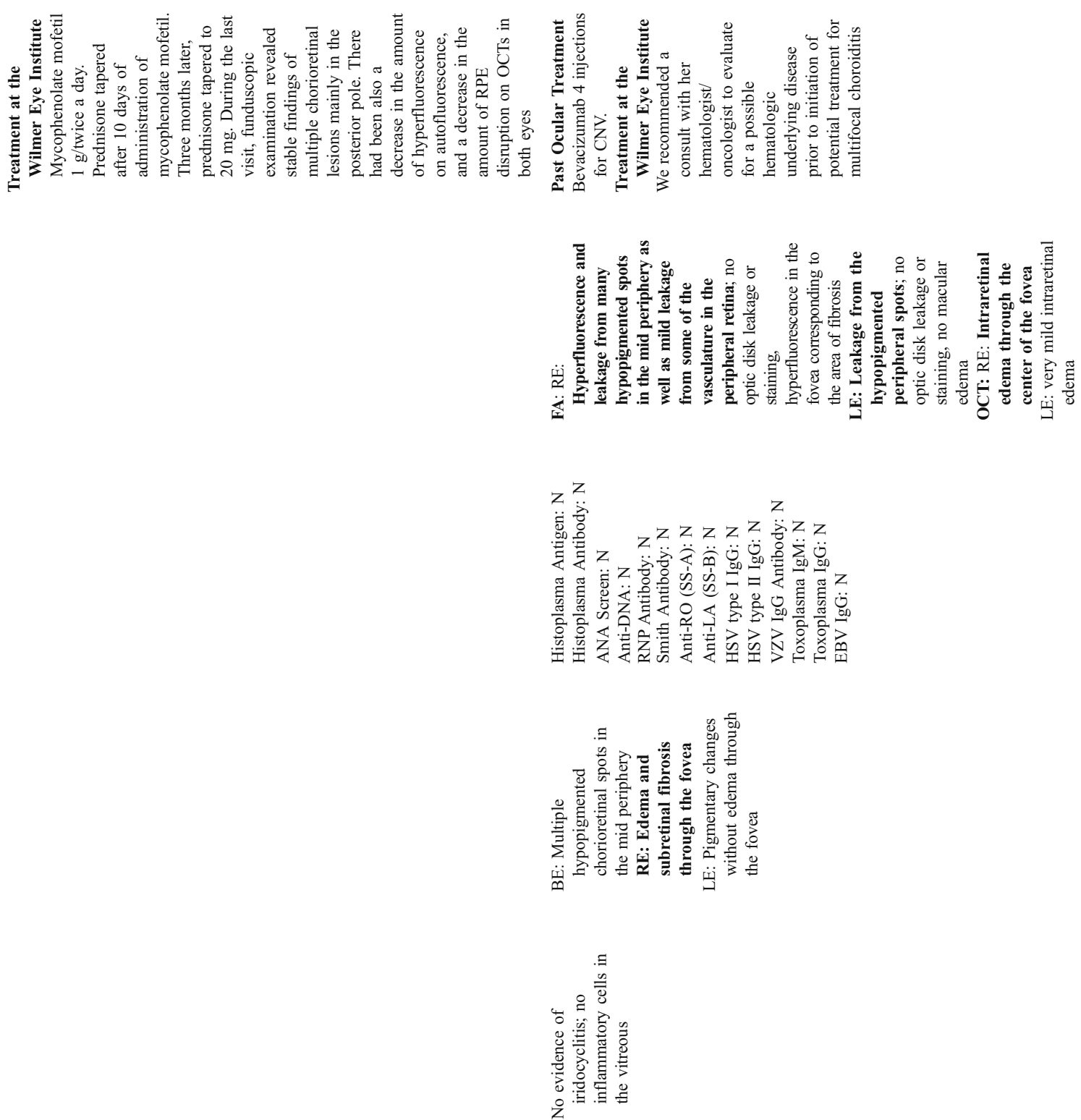

$\stackrel{\infty}{=}$

ํํㄴ

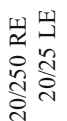

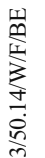




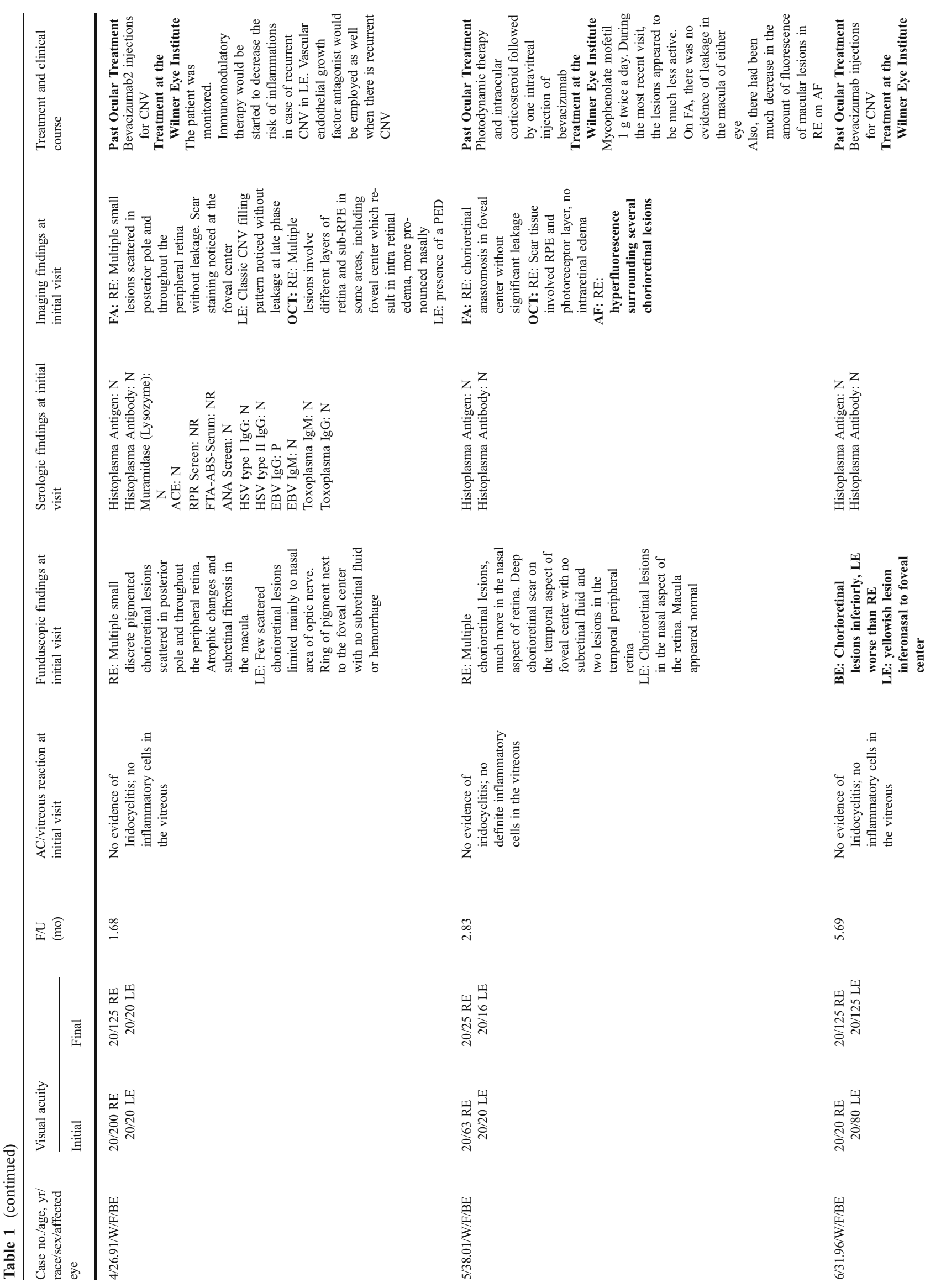




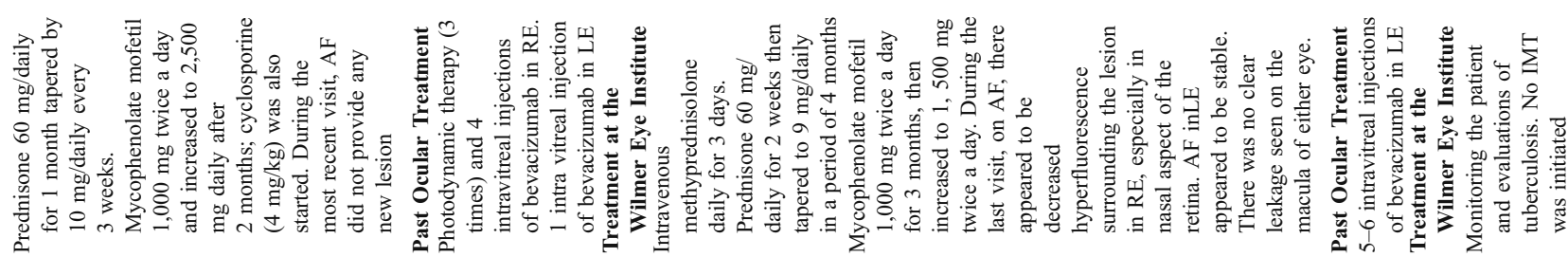
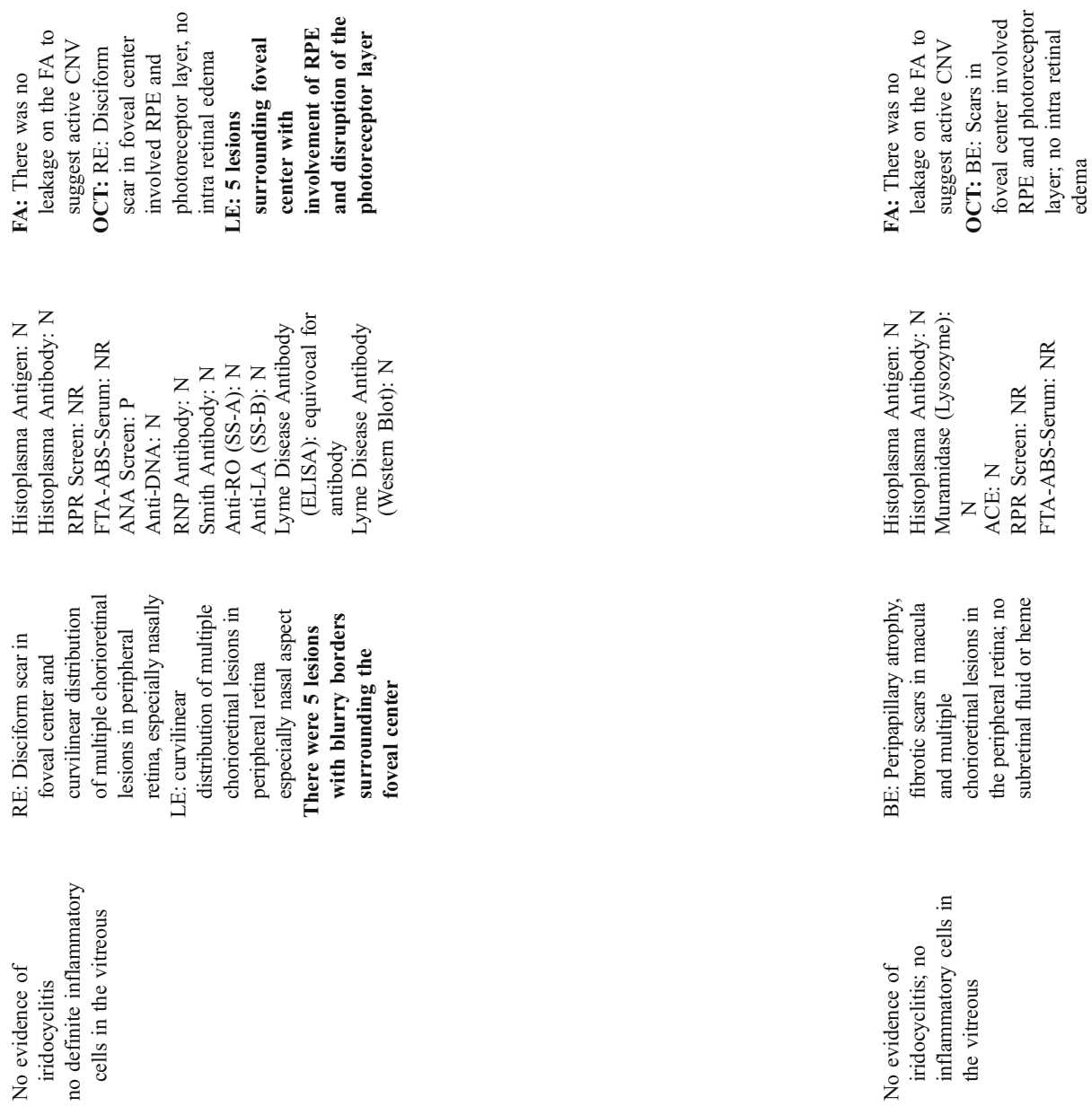

กิ่

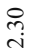

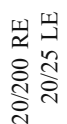

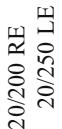

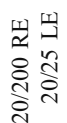

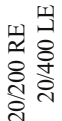

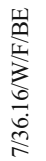

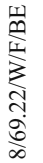




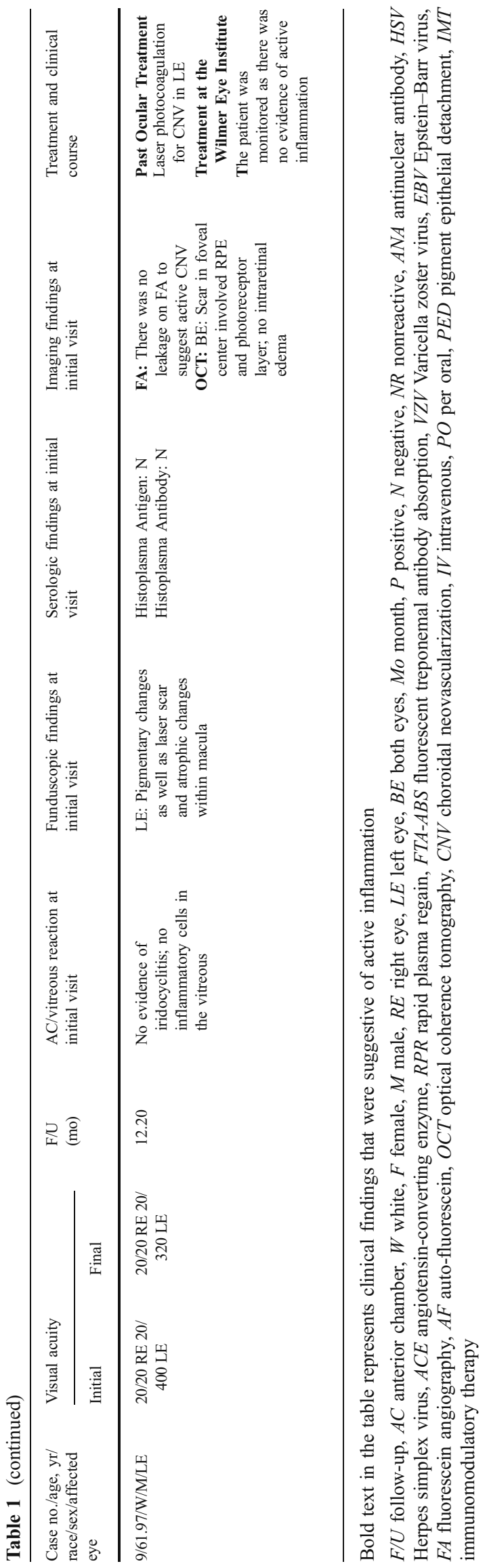

those seen in OHS were present in our patients. However, the chorioretinal lesions resulted from inflammatory processes may be similar to those in OHS, with pigmented borders and centers.

An enlarged blind spot at the time of presentation with acute lesions or thereafter when the lesions are inactive has been reported in patients with MFC [3]. For patient 5, the Amsler visual testing revealed the presence of an old scotoma in the nasal aspect of the field as well as a newer small scotoma in the temporal aspect of the right visual field. Multifocal chorioretinal lesions were seen in every patient. The lesions were generally smaller and more numerous than the typical histoplasma spots in OHS [4, 5]. There was a predominance of women similar to other reports on MFC [1, 2]. The histoplasma antigen/antibody, performed in the same laboratory was negative in all our patients. Previously, serologic testing for Histoplasma capsulatum used to have relatively low sensitivity with positive result in $15 \%$ of the patients [2]. The recently available serologic testing for $H$. capsulatum antigen is highly sensitive. If it is disseminated histoplasmosis in an immunocompetent patient, the sensitivities in serum and urine samples are $82 \%$ and $92 \%$, respectively; specificity is $98 \%$ and its reproducibility is excellent [6]. In those with limited pulmonary disease, the sensitivities in serum and urine samples are $68.6 \%$ and $64.6 \%$, respectively [7]. In antibody test against $H$. capsulatum, the positive immunodiffusion reactions involve one or more specific precipitin bands. Of these bands, the first to appear in active histoplasmosis is the " $\mathrm{M}$ " band, which is seen in approximately $70 \%$ of proven cases. The $\mathrm{M}$ band is also seen in a number of patients with past infections and in $20 \%$ of those with recent Histoplasma skin testing. The " $\mathrm{H}$ " band is usually seen in active and progressive histoplasmosis and almost always exists in the presence of the " $\mathrm{M}$ " band, although it is found less often in approximately $10 \%$ of proven cases (Quest Diagnostics Nichols Institute, 14225 Newbrook Drive, Chantilly, VA 20153, and Immuno Mycologics, Inc (Immy) [8]).

Evidence of Epstein-Barr virus (EBV) infection was detected in one of our patients, which is consistent with Tiedeman's report [9] about the possible relationship between MFC and recent EBV infection.

The lesions observed in patients in the index study were also different from those in PIC, which typically do not have inflammatory signs, cells, or vitreous inflammation, and often constitute a cluster of macular lesions [1]. PIC patients have chorioretinal scars mostly in the posterior pole and the clinical course is less likely complicated by recurrent inflammation. The visual prognosis is usually stable unless CNV develops. Unlike PIC, patients with MFC have chorioretinal scars mostly in the mid-peripheral retina and a course characterized by waxing and waning inflammation that results in poor visual prognosis [10]. 
The imaging findings in the index study supported the MFC lesions and were consistent with the lesions described as MFC in other studies [11, 12]. Retinal pigment epithelium (RPE) was altered on OCT images. The hyper-fluorescent leakage on FA images as well as hypo-fluorescence on autofluorescene was consistent with previous descriptions of MFC. These spots were associated with chorioretinal scars and atrophy or absence of RPE cells detected in the posterior pole of affected eyes [11, 12].

Once the patients were thought to have MFC with recurrent diseases, corticosteroids and IMT were initiated, which led to full control (no recurrences) of inflammation and preservation of vision secondary to absence of active inflammation [13]. IMT also allowed tapering of dosages of corticosteroids to avoid long-term complications.

The current study emphasizes the importance of considering the possibility of other diagnoses when the disease has atypical characteristics and behavior. Based on our experience, when the actual chorioretinal lesions are inflamed in the presence of an inactive $\mathrm{CNV}$, the diagnosis of OHS might be in doubt and should be reconsidered, and different treatment approach might be required.

However, we also recognize the many limitations of our study. The index study is a retrospective review of a small number of patients. Many of the patients were followed for only a short period of time. Longer follow-up time in a large group of patients will provide more information regarding the challenges in the diagnosis and management of MFC masquerading as other conditions such as OHS and will also help to confirm that treatment with IMT decreases recurrences of diseases, as expected in MFC, and not in OHS. We also recognize that many patients might not have presented with appropriate information and history to aid the referring physicians to consider MFC as possible etiologies.

Differentiation between MFC and OHS can be quite challenging, especially when both conditions are quiescent and there has been no previous examination or documentation of the appearance of the fundus [1]. Serologic testing for $H$. capsulatum, which is recently available with high sensitivity, should be employed to aid in the management of such patients, as absence of histoplasma titer and clinical characteristics of recurrent MFC may be more supportive for a diagnosis of (inflammatory) MFC and may lead to consideration of IMT and other appropriate treatments for MFC.
Conflict of Interest The authors declare that they have no conflict of interest.

Open Access This article is distributed under the terms of the Creative Commons Attribution License which permits any use, distribution and reproduction in any medium, provided the original author(s) and source are credited.

\section{References}

1. Parnell JR, Jampol LM, Yannuzzi LA, Gass JD et al (2001) Differentiation between presumed ocular histoplasmosis syndrome and multifocal choroiditis with panuveitis based on morphology of photographed fundus lesions and fluorescein angiography. Arch Ophthalmol 119:208-212

2. Dreyer RF, Gass DJ (1984) Multifocal choroiditis and panuveitis. A syndrome that mimics ocular histoplasmosis. Arch Ophthalmol 102:1776-1784

3. Khorram KD, Jampol LM, Rosenberg MA (1991) Blind spot enlargement as a manifestation of multifocal choroiditis. Arch Ophthalmol 109:1403-1407

4. Smith RE, Ganley JP, Knox DL (1972) Presumed ocular histoplasmosis. II. Patterns of peripheral and peripapillary scarring in persons with nonmacular disease. Arch Ophthalmol 87:251257

5. Deutsch TA, Tessler HH (1985) Inflammatory pseudohistoplasmosis. Ann Ophthalmol 17:461-465

6. Garringer TO, Wheat LJ, Brizendine EJ (2000) Comparison of an established antibody sandwich method with an inhibition method of Histoplasma capsulatum antigen detection. J Clin Microbiol 38 (8):2909-13

7. Swartzentruber S, Rhodes L, Kurkjian K, Zahn M, Brandt ME, Connolly P, Wheat LJ (2009) Diagnosis of acute pulmonary histoplasmosis by antigen detection. Clin Infect Dis 49(12):187882

8. http://www.immy.com/?q=histoplasma

9. Tiedeman JS (1987) Epstein-Barr viral antibodies in multifocal choroiditis and panuveitis. Am J Ophthalmol 103:659-663

10. Kedhar SR, Thorne JE, Wittenberg S, Dunn JP et al (2007) Multifocal choroiditis with panuveitis and punctate inner choroidopathy: comparison of clinical characteristics at presentation. Retina 27:1174-1179

11. Haen SP, Spaide RF (2008) Fundus autofluorescence in multifocal choroiditis and panuveitis. Am J Ophthalmol 145:847-853

12. Yasuno Y, Okamoto F, Kawana K, Yatagai T et al (2009) Investigation of multifocal choroiditis with panuveitis by threedimensional high-penetration optical coherence tomography. J Biophotonics 2:435-441

13. Michel SS, Ekong A, Baltatzis S, Foster CS (2002) Multifocal choroiditis and panuveitis: immunomodulatory therapy. Ophthalmology 109:378-383 